\title{
ERECTOR SPINAE PLANE BLOCK FOR BREAST CANCER SURGERY. A CASE SERIES.
}

\section{P. Alcántara 1, M. Pérez Poquet 1, E. Vallejo Tarrat 2, I. Barco Nebreda 2 , C. Pérez Torrentó 1. \\ 1. Hospital Universitari Mútua Terrassa, Department of Anaesthesiology \& Pain Medicine, Terrassa, Spain. \\ 2. Hospital Universitari Mútua Terrassa, Department of Gynecology \& Obstetrics, Terrassa, Spain.}

\section{BACKGROUND AND AIMS}

Breast cancer is the most common cancer among women and surgery is its main treatment.

Morbidities associated to that surgery are acute postoperative pain and postoperative nausea and vomiting (PONV).

New techniques of regional anaesthesia have been described for thoracic wall surgeries in order to relief the pain, being erector spinae plane (ESP) block one of them.

Our aim is to evaluate postoperative pain using VAS score in breast cancer surgery (BCS) by performing ESP block.

We also recorded opioid consumption, incidence of PONV and length of stay (LOS).

\section{METHODS}

- Retrospective analysis

- October 2017 - April 2018

- $\mathrm{n}=16$ patients

- Surgeries carried out:

- Tumorectomy + lymph node biopsy $(n=13)$

- Mastectomy $(n=3)$

- General anaesthesia + ESP Block

- Intraoperative analgesia: paracetamol + dexketoprophen

- Antiemetic prophylaxis: dexamethasone + ondansetron

\section{ESP BLOCK}

- Awake patient on the seated position

- Ultrasound guided

- 13-6 MHz probe

- T5 transverse process

- $50 \mathrm{~mm}$ needle

- Single puncture

- Levobupivacaine $0.3 \%(30 \mathrm{~mL})$

Fig. 1: Anatomical references to perform the block. $\mathrm{ML}=$ Midline. TP $=$ Transverse Process. ${ }^{2}$
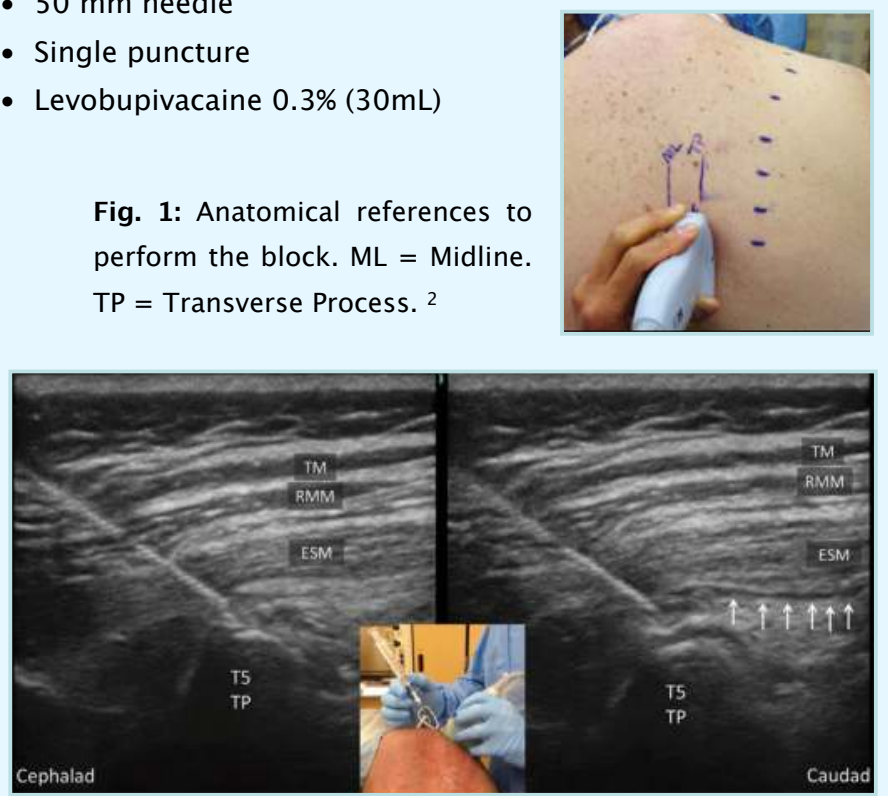

Fig. 2: Probe in LAX and IP needle insertion with the tip on T5 TP. Arrows $=$ spread of local anaesthetic, $\mathrm{TM}=$ Trapezius Muscle, RMM $=$ Romboid Major Muscle, ESM = Erector Spinae Muscles. ${ }^{1}$
RESULTS

at PACU

after PACU

$24 \mathrm{~h}$ after surgery

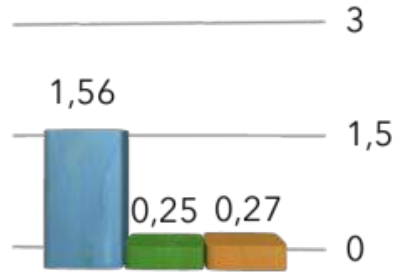

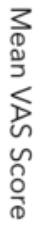

Time of VAS score record

Fig 4. Postoperative pain at Post-Anaesthesia Care Unit (PACU), after PACU and $24 \mathrm{~h}$ after surgery.

IV Tramadol ( $\mathrm{mg} / \mathrm{kg})$

IV Morphine
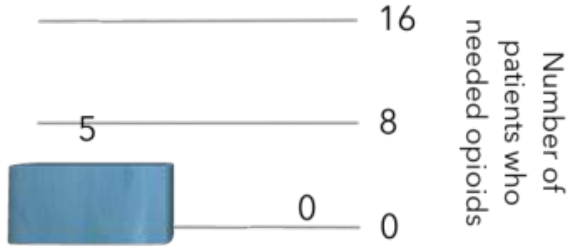

Type of opioid

Fig 5. Opioid consumption after surgery.

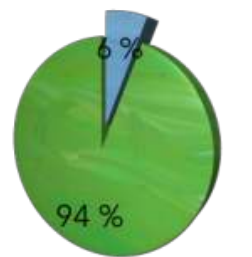

Patients with PONV

Patients without PONV

Fig 6. PONV incidence.

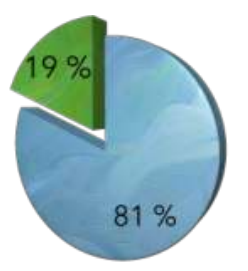

1 day of LOS

More than 1 day of LOS

Fig 7. Length of stay (LOS).

\section{CONCLUSIONS}

Postoperative VAS scores in patients who underwent ESP block were low enough to think that ESP block provides good postoperative analgesia on BCS.

Patients rarely needed opioids, had low incidence of PONV and short LOS. Nevertheless, further research is needed.

\section{REFERENCES}

1. Forero $\mathrm{M}$ et al, The erector spinae plane block: a novel analgesic technique in thoracic neuropathic pain. Reg Anesth Pain Med 2016; 41: 621-7.

2. Chin, K. J. et al, The analgesic efficacy of pre-operative bilateral erector spinae plane (ESP) blocks in patients having ventral hernia repair. Anaesthesia, 72(4), 452-460. 